How does Interhemispheric Communication in Visual Word Recognition Work? Deciding between Early and Late Integration Accounts of the Split Fovea Theory.

\author{
Lise Van der Haegen ${ }^{1,2}$ Marc Brysbaert ${ }^{1}$ Colin J. Davis ${ }^{1}$ \\ ${ }^{1}$ Royal Holloway, University of London \\ ${ }^{2}$ Ghent University, Belgium
}

Address: $\quad$ Marc Brysbaert

Royal Holloway, University of London

Department of Psychology

Egham TW20 0EX

United Kingdom

Tel. +441784443526

e-mail: marc.brysbaert@rhul.ac.uk 


\begin{abstract}
It has recently been shown that interhemispheric communication is needed for the processing of foveally presented words. In this study, we examine whether the integration of information happens at an early stage, before word recognition proper starts, or whether the integration is part of the recognition process itself. Two lexical decision experiments are reported in which words were presented at different fixation positions. In Experiment 1, a masked form priming task was used with primes that had two adjacent letters transposed. The results showed that although the fixation position had a substantial influence on the transposed letter priming effect, the priming was not smaller when the transposed letters were sent to different hemispheres than when they were projected to the same hemisphere. In Experiment 2, stimuli were presented that either had high frequency hemifield competitors or could be identified unambiguously on the basis of the information in one hemifield. Again, the lexical decision times did not vary as a function of hemifield competitors. These results are consistent with the early integration account, as presented in the SERIOL model of visual word recognition.
\end{abstract}




\section{How does Interhemispheric Communication in Visual Word Recognition Work? Deciding between Early and Late Integration Accounts of the Split Fovea Theory.}

A fundamental feature of the human brain is that it is divided in two cerebral hemispheres. This anatomical organization, together with the fact that the optic tracts are partially decussated in the optic chiasm, has extensive consequences for visual perception: Visual stimuli that are presented in the left visual field (LVF) are initially projected to the visual cortex of the right hemisphere $(\mathrm{RH})$, whereas information in the right visual field (RVF) is sent to the left cerebral hemisphere (LH) (Leff, 2004).

There is more uncertainty about what happens at the center of the visual field. In particular, there has been disagreement about what happens in the fovea, the area of 1 to 3 degrees of visual angle in the center of the retina. It was believed for a long time that foveally presented stimuli were projected bilaterally to both brain halves (e.g., Bunt, Minckler, \& Johanson, 1977). However, recent reviews have shown that most evidence is in line with a split fovea view (e.g., Brysbaert, 1994, 2004; Lavidor \& Walsh, 2004a). Numerous anatomical (e.g., Tootell, Switkes, Silverman, \& Hamilton, 1988), functional (e.g., Lavidor, Ellison \& Walsh, 2003; Lavidor \& Walsh, 2004b; Martin, Thierry, Démonet, Roberts, \& Nazir, in press; Portin, Salenius, Salmelin, \& Hari, 1998; Reinhard \& Trauzettel-Klosinski, 2003) and behavioral studies (e.g., Brysbaert, 1994; Brysbaert, Vitu, \& Schroyens, 1996; Ellis, 2004; Ellis, Brooks, \& Lavidor, 2005; Hunter, Brysbaert, \& Knecht, 2007; Lavidor, Ellis, Shillcock, \& Bland, 2001; Lavidor, Hayes, Shillcock, \& Ellis, 2004) have provided data in line with the split fovea view and its implications for visual word recognition.

One behavioral approach that has been used to investigate this issue is to look at the effects of brain laterality on the Optimal Viewing Position (OVP) effect, an effect that arises from the visual constraints in word processing (O’Regan \& Jacobs, 1992). In this paradigm participants are asked to process words presented in such a way that the participants are seeing the words at different letter positions. On some trials the words are presented such that the first letter is at the fixation location, on other trials the words are presented such that the second letter is at the fixation location, and so on (see Figure 2 for an example of the use of the technique in the present paper). The usual finding is that participants are fastest to process a word when they fixate between the beginning 
and the center of the word. This is the so-called optimal viewing position for word recognition. Word processing times increase when the participants have to fixate at other positions in the word, in particular when they have to fixate towards the end of the word (Figure 1).

In a review article, Brysbaert and Nazir (2005) argued that the optimal viewing position originates from four factors. First, because of the drop of visual acuity outside the fixation location, the visibility of letters decreases the further away they are from the fixation position. This factor alone would predict an OVP in the middle of the word and similar processing costs for fixations towards the beginning and the end of the word (i.e., the OVP curve would be U-shaped). Three more factors explain why processing times are faster for fixations on the first half of the word than for fixations on the last half (giving the OVP-curve a J-shape rather than a U-shape). The first of these extra factors is that the first letters of a word usually are more informative than the letters at the other positions, giving an advantage to fixations on the word beginning. The second extra factor is that in normal reading the eyes usually land left of the center of the words, so that the visual system is more practiced in processing words from this particular position. Finally, a fixation on the intial letters of a word makes the larger part of the word fall in RVF, whereas a fixation on the end letters makes the larger part of the word fall in LVF. Given that most people are left dominant for language processing, they have an advantage for verbal information presented in RVF unless, of course, the fovea projects information bilaterally.

The analysis of the OVP effect suggests a straightforward way to test the split fovea assumption. If the fovea is split, then participants with left language dominance should have an advantage for fixations on the beginning of a word (so that the word falls in RVF), whereas participants with right language dominance should have an advantage for fixations on the end of a word. In contrast, a bilateral representation of the fovea predicts no effect of cerebral dominance on the OVP-curve as all the information is sent to both hemispheres simultaneously. This test was reported by Hunter et al. (2007). They assessed the language dominance of participants for speech production by means of functional transcranial Doppler sonography (fTCD) and fMRI. On the basis of these data, a group of left dominant and a group of right dominant participants were distinguished. Thereafter, a naming task was administered, involving three-, five-, and 
seven-letter German words. Participants were asked to fixate a gap between two vertically aligned lines in the middle of the screen, which, similar to the paradigm used by O'Regan and Jacobs (1992), served as the fixation point (see also Figure 2 below). Throughout the experiment, the words were presented at each possible letter fixation location, by shifting their position horizontally across the screen. The results were clearcut: as predicted, the OVP changed as a function of the language dominance of the participants: Participants with left hemisphere dominance showed a stronger wordbeginning superiority effect than right-dominant participants.

Insert Figure 1 about here

The fact that the OVP curves differ for participants with left and right cerebral dominance is strong evidence that interhemispheric communication is needed for foveal word recognition. This raises the question how such integration is achieved. There are two main possibilities, which we shall refer to as the early integration account and the late integration account.

According to the early-integration account, interhemispheric transmission of information occurs early on in visual word processing, before word recognition proper starts. Information about the stimulus is transferred from the non-dominant hemisphere to the dominant hemisphere, where word recognition takes place (Brysbaert, 1994). This hypothesis is explicitly incorporated in the SERIOL model of visual word recognition (Whitney, 2001). One of the basic assumptions of this model is that visual word recognition happens serially, from the word beginning to the word end. To achieve this, the word recognition system has to overcome two characteristics of the perceptual input. The first is that letters close to the fixation location have a greater activation than letters further away from the fixation location due to the decrease of visual acuity outside central vision. This is a problem for the letters to the left of the fixation position, because the first letter of the word is further away from the fixation position than the second letter, which in turn is further away than the third letter, and so on. As a consequence, the retina-based activation of the first letter will be lower than 
that of the second letter, which is lower than that of the third letter, and so on until the fixation location is reached. In order to achieve left-right seriality, it is therefore necessary to invert the initial retina-based activation gradient of the letters to the left of the fixation location, so that the activation of the first letter becomes stronger than that of the second letter, etc.

The second feature that must be overcome is the splitting of the word between the left and the right hemisphere. In particular, the fact that the word beginning (which falls in the LVF for a centrally fixated word) is sent to the non-dominant $\mathrm{RH}$ is a problem. Therefore, the SERIOL model assumes that for leftdominant readers the information about the word end in LH is inhibited until the information about the word beginning has been transferred from RH to LH. In other words, according to the SERIOL model, word recognition does not start until the activation of the first letter of the word has reached a threshold value in the dominant hemisphere.

Cai, Lavidor, Brysbaert, Paulignan, and Nazir (2008) reported a study that supports the early-integration view of the split fovea theory. Like Hunter et al. (2007), they first assessed the laterality of the frontal word production brain areas in French readers by means of a verb generation task. Next, they measured the occipito-temporal processes involved in visual word recognition with the use of a parafoveal word reading task. The latter task had been introduced by Cohen, Dehaene, Naccache et al. (2000) to show that in left dominant participants a brain region in the left occipito-temporal sulcus is involved in word processing, independently of whether the word is shown in RVF or LVF. Cohen et al. called this area "the visual word form area" and claimed that it was the first language-specific area needed for reading orthographic stimuli. Other research confirmed the left lateralization of this visual word form area in righthanded participants, both in alphabetical languages (Vigneau, Jobard, Mazoyer, \& TzourioMazoyer, 2005) and in non-alphabetical languages such as Chinese (Liu et al., 2008). Cai et al. (2008) replicated this finding for participants with left frontal language dominance and, in addition, showed that the area was lateralized to the right hemisphere for participants with right frontal language dominance. This is in line with the hypothesis of a rapid convergence of information about visually presented words in the language-dominant hemisphere and can thus serve as a first indication for the correctness of the early integration account. 
A more radical view of the role of interhemispheric transfer in visual word recognition is the late-integration account, proposed by Shillcock, Ellison and Monaghan (2000) ${ }^{1}$. They claim that word recognition occurs intrahemispherically in the first stages of processing. This means that both hemispheres start processing the word on the basis of the visual input they receive, and integrate the information only at a relatively late stage. As a consequence, different fixation locations within the target word provide the brain with different information about the word. Word recognition is fundamentally different after fixation on the first letter, the middle letter, or the last letter. Fixation on the first letter means that the whole word falls in the RVH, and therefore is processed exclusively by the LH. In contrast, fixation on the last letter results in the RH taking the burden for word recognition. Fixation at the middle of a word sends partial information to both brain halves, which independently activate candidate word representations. Shillcock et al. (2000) further hypothesized that it is not needed to code all letter positions of a word. $99.8 \%$ of the words in the lexicon they used could be identified on the basis of the outer letters and the two letters surrounding the fixation position. Finally, a third assumption of Shillcock et al. (2000) was that the brain halves have different characteristics with respect to letter encoding, with the nondominant hemisphere using coarser coding than the dominant hemisphere.

Evidence supporting the late-integration account of the split fovea theory can be found in Pernet, Uusvuori, and Salmelin (2007). In this study, the mechanisms of word priming were examined in conditions where both the prime and the target were presented in the foveal area (foveal priming), and in conditions where the prime was presented parafoveally in LVF or RVF, followed by the target word in foveal vision (parafoveal-on-foveal priming). The behavioral data revealed a priming effect at all prime-to-target delays in the case of foveal priming, but parafoveal-on-foveal priming was only observed at a delay of $50 \mathrm{~ms}$. In addition, the magnetoencophalography (MEG) part of Pernet et al.'s (2007) study showed that there were differences between the two types of priming at the neural level: parafoveal priming was strongest in the contralateral occipito-temporal, temporal and fronto-temporal regions, whereas the

\footnotetext{
${ }^{1}$ Note: Throughout this study, we will use the term split fovea to refer to the assumption that centrally fixated stimuli are split between the left and the right hemisphere and we will describe the split fovea theory proposed by Shillcock et al. (2000) as the model of Shillcock et al. (2000).
} 
processing of the foveal priming activated areas in the fronto-temporal and temporal regions of both hemispheres (with a significantly stronger effect in LH than in RH though).

The aim of the current study was to investigate which account of the split fovea theory best explains behavioral word recognition data. One way to make a decision between the early and the late integration accounts is to examine how word processing differs as a function of the fixation location. If the early integration account is correct, then fixation location should have no effect on which word candidates become activated and compete with one another during the identification process, as all the information is first converged in the dominant hemisphere. Thus, this view does not predict an interaction between the fixation position and the information distribution within the word that is presented. In contrast, according to the late integration account, fixation location will have an effect on the activation of other word candidates. This view predicts that word processing will be particularly disrupted when the information received by each hemisphere activates word representations that are incompatible with the target word.

Two experiments were conducted, each testing different predictions that distinguished between the early and late accounts. The first experiment used a lexical decision task with masked priming. It investigated whether the transposed letter priming effect (Perea \& Lupker, 2003) is diminished when the transposed letters are sent to different hemispheres. According to the early integration account no interaction should be found between the fixation location and the transposed letter pair, as all the information is transferred to the dominant hemisphere before word recognition starts. In contrast, the late integration account predicts smaller priming effects for primes with transposed letters when these letters are sent to different hemispheres compared with when they are sent to the same hemisphere, because in the former case the information of the prime received by each hemisphere is much less informative about the upcoming target word (see below).

The second experiment, also a lexical decision task with stimuli presented at different fixation locations, examined the influence of what will be referred to as 'hemifield competitors'. If part of the target word has a higher-frequency hemifield competitor, then the late integration account predicts that, in the initial stages of visual 
word processing, the receiving hemisphere will co-activate the higher frequent word that is compatible with the given information, because each hemisphere independently activates word candidates on the basis of the information they receive. As a result, this theory predicts competition effects when each word half strongly suggests words other than the target. The early integration view predicts that ambiguity of the word halves will not have an effect on the reaction times in a lexical decision task.

\section{Experiment 1}

In a masked priming experiment Perea and Lupker (2003) found that lexical decision latencies were faster for targets preceded by transposed letter (TL) primes than for the same targets preceded by replacement letter (RL) control primes. Thus participants were faster to decide that JUDGE was an existing English word, when immediately before the TL prime jugde was presented briefly than when immediately before the RL prime jupte was presented. Even faster latencies were found when the targets were preceded by identity primes (i.e., judge - JUDGE). These findings indicate that letter transposition is to some extent disruptive but less so than letter change, suggesting that letter positions in visual words are less strictly encoded than letter identities, in line with the second assumption of the Shillcock et al. (2000) model (see above; for other evidence about TL priming see Forster, Davis, Schoknecht, \& Carter, 1987; Schoonbaert \& Grainger, 2004).

The present experiment goes beyond Perea and Lupker (2003) by systematically varying the fixation position relative to the transposed letter pair. The critical condition is the one in which the letter string is fixated between the transposed letters (e.g., graden - GA-RDEN; the hyphen indicates the fixation position). If Shillcock et al.'s (2000) model is correct, then primes in which the transposed letters are sent to different hemispheres should cause significantly less priming than when they are sent to the same hemisphere (as in gra-den - GAR-DEN). This is because in Shillcock et al.'s model the letter positions within a hemisphere are less strictly encoded than those between hemispheres (due to the split of the information between $\mathrm{LH}$ and $\mathrm{RH}$ ). As a result, the lexical representations of GARDEN in LH and RH will be activated more on the basis of the input gra-den than on the basis of the input gr-aden (in the former case, the 
information of the prime and the target in RVF is compatible, and the information in LVF is also compatible as far as the letter identities are concerned; in the latter case no prime hemifield information is compatible with the target). In contrast, if the early integration account is correct, there should be no difference in the TL priming effect for fixation positions between the transposed letters and fixation positions next to the transposed letters, because word recognition proper does not commence until all of the letters have been assembled in the dominant hemisphere.

Method

\section{Participants}

Twenty-eight native English speaking students from Royal Holloway, University of London participated in the experiment. Twenty-three participants were female. All had normal or corrected-to-normal vision, did not have any reading problems, were unaware of the purpose of the experiment and were paid for their participation. All participants but two were righthanded. ${ }^{2}$

\section{Stimuli}

The stimulus sample consisted of 600 target words, 600 nonwords, and 30 fixation control digits. The words were selected from the English Lexicon Project (Balota, Cortese, Hutchison, et al., 2002) and the CELEX database, using the Wordgen software (Duyck, Desmet, Verbeke, \& Brysbaert, 2004). All had a word length of six letters, were monomorphemic and were controlled for frequency (minimum 1 per million), grammatical class (adjectives, substantives or verbs) and orthographic neighborhood (maximum three neighbors). The nonwords were constructed by changing one or two letters at different positions of words that had to be excluded due to the previously described controls.

\footnotetext{
${ }^{2}$ About one quarter of lefthanded students have right hemisphere dominance or bilateral language representation (Hunter \& Brysbaert, 2008; Knecht et al., 2000). By comparing the OVP curves, we could ascertain that this was not the case for the two left-handed participants included in the present study. In addition, we verified that all conclusions remained valid when these two participants were excluded from the analyses.
} 
The targets were presented on the screen in uppercase and were preceded by lowercase primes. These six-letter primes were (a) transposed letter nonwords (TL primes) created by transposing the second and third letter of each target word (e.g., tewlve - TWELVE), (b) replacement letter nonwords (RL control primes) created by replacing the letters at the second and third position of the target words (e.g., tuslve TWELVE), (c) TL primes created by transposing the fourth and fifth letter of each target word (e.g., twevle - TWELVE), (d) RL control primes created by replacing the letters on the fourth and fifth position of the target words (e.g., twemde - TWELVE). The TL primes and RL control primes were matched with respect to word-shape, consonant-vowel structure and mean log bigram and trigram token frequency $(t \mathrm{~s}<1$, see Table 1). The constructed primes had no orthographic neighbors or deletion neighbors, and were not pseudohomophones nor transposed-letter words themselves. The estimates for this matching process were acquired by using the N-Watch software (Davis, 2005). TL and RL control primes were also constructed for the target nonwords.

Insert Table 1 about here

Each target word and nonword thus had four different primes. Moreover, each prime-target pair could be presented at five different fixation positions (i.e. between letter positions 1-2, 2-3, 3-4, 4-5, and 5-6, see Figure 2). These fixation conditions ensured that any effects of interest were not simply due to differences in visual acuity. If this were the case, then priming effects should be gradually increasing as the distance between the fixation position and the transposed letters increases. The full list of stimuli used in the experiment can be found in the Appendix appended to the electronic version of this article.

Insert Figure 2 about here 
Design

As described in the above paragraph, the experiment included three factors: type of prime (TL or RL control prime), position of transposition (positions 2 and 3, or 4 and 5), and fixation location (after the first, second, third, fourth, or fifth letter, as indicated by the vertical lines in the examples in Figure 2). This resulted in a $2 \times 2 \times 5$ factorial design.

Due to the fact that the target words and nonwords appeared in 20 different conditions ( 4 primes and at 5 fixation positions), the stimuli were divided into 20 lists. Each list contained all target words and nonwords, equally distributed across the 20 conditions. Thus, 30 stimuli per condition were presented to each participant. The words and nonwords were assigned to the lists according to a Latin square design, in order to avoid any stimulus being presented more than once in the same list.

\section{Procedure}

Stimuli were presented in Courier New font, size 12, on a CRT display, using the DMDX software (Forster \& Forster, 2003). Participants were sitting at a viewing distance of approximately $60 \mathrm{~cm}$.

At the beginning of the lexical decision task, a forward mask consisting of ten hash marks was presented together with the appearance of the two vertical lines. The mask covered all possible positions of the following primes and targets, so that the participants were unable to know at which position the stimulus would appear. They were asked to fixate the gap between the two vertically aligned lines as soon as the lines appeared. The fixation lines remained in the center of the screen for the total duration of the trial. After $500 \mathrm{~ms}$, the forward mask was replaced by the prime for $57 \mathrm{~ms}$, immediately followed by the target. Participants were instructed to press the right button if the target was a word and the left button if it was a nonword (the response matching was reversed for two left-handed participants). The next trial started $1 \mathrm{~s}$ after the response to the previous trial. The reaction times and the accuracy of the trials were registered by means of a response box. Reaction time (RT) measurement started at the onset of the target word. Targets were displayed until a response was made. 
To ensure that the fixation instruction was adhered to, at random intervals a digit trial was presented instead of a word trial (for a total of 30 trials). On the digit trials, the forward mask was replaced by a digit for $80 \mathrm{~ms}$. The digit was presented in the gap between the fixation lines and was followed by a mask consisting of an ASCII code 35 (\#). Participants had to indicate whether the digit was even (dominant hand) or odd (non-dominant hand). The experimenter told the participants that the correct evaluation of the digits was of high importance for the results of the experiment.

The actual experiment was preceded by eleven practice trials, consisting of one number, five word stimuli and five nonword stimuli, which did not return in the experimental phase. The experiment was run in two sessions of approximately 30 minutes. In each session, there were three breaks provided. There was a minimum delay of one day between the two sessions.

\section{Results}

The inclusion of digit trials to control for adequate fixation turned out to be necessary. Of the first 15 participants tested, 8 made more than $20 \%$ errors, making us unsure about their fixation position at the onset of the prime. In order to redress this problem, subsequent participants were given a greater cash incentive for good performance on the digit classification task. From that moment on, there were no more outliers and the overall percentage of correct digit classification for the 20 participants included in the design was $91.2 \%$.

Incorrect responses ( $4.7 \%$ of the data for word targets) and latencies that were shorter than $300 \mathrm{~ms}$ or longer than $2000 \mathrm{~ms}$ ( $0.4 \%$ of the data for word targets) were excluded from all reaction time analyses. One more participant had to be excluded, because of a low level of accuracy (16.3\% errors) and high mean reaction time (736 $\mathrm{ms}$ ), relative to the nineteen remaining participants. Analyses of Variance (ANOVAs) were carried out by subjects (F1), but not by items (F2), because our stimulus list contained all available words and because we used a counterbalanced design (Raaijmakers, Schrijnemakers, \& Gremmen, 1999). 
Prime type (TL prime vs. RL prime), position of transposition (2-3 vs. 4-5) and fixation location (first, second, third, fourth and fifth) were treated as repeated factors and participants as the random variable in the analysis.

Insert Table 2 about here

Table 2 shows the mean response times and error rates for the target words. The ANOVA of the mean RTs revealed a main effect of prime type $[F 1(1,18)=41.09, M S E$ $=2,256.99, p<.001$ ], with faster responses to targets with TL primes (mean $593 \mathrm{~ms}$ ) than with RL primes (mean $625 \mathrm{~ms}$ ). ${ }^{3}$ The main effect of fixation location was also significant $[F 1(3.23,58.16)=23.01, M S E=2,179.71, p<.001]$, with mean reaction times of $609 \mathrm{~ms}, 590 \mathrm{~ms}, 590 \mathrm{~ms}, 608 \mathrm{~ms}$ and $647 \mathrm{~ms}$ for the targets at the first, second, third, fourth and fifth fixation location respectively. Finally, the three-way interaction between prime type, position of transposition, and location of fixation was also significant $[F 1(3.15,56.60)=8.76, M S E=881.54, p<.001]$. The analysis yielded no other significant effects (all $p \mathrm{~s}>.05$ ).

The ANOVA of the mean error rates revealed a main effect of prime type $[F 1(1,18)=6.47, M S E=.003, p<.05]$, with lower error rates in the TL prime condition (mean $4.2 \%$ ) compared to the RL prime condition (mean 5.6\%). Fixation location had also a significant main effect $[F 1(3,53.91)=4.72, M S E=.003, p<.01]$, with mean error rates of $4.8 \%, 4.0 \%, 3.8 \%, 5.3 \%$ and $6.6 \%$ for the conditions of the first, second, third, fourth and fifth fixation location respectively. The three-way interaction between prime type, position of transposition, and fixation location was significant $[F 1(3.21,57.82)=4.73, M S E=.002, p<.01]$. Other effects of error rates were not significant (all $p s>.26)$.

Insert Figure 3 about here

\footnotetext{
${ }^{3}$ Analysis of the confidence interval (Masson \& Loftus, 2003) indicated that all TL priming effects larger than $23 \mathrm{~ms}$ are significant at the .05 level.
} 
To further investigate which factors contributed to these effects, two linear regression analyses were performed with the TL priming effects as the dependent variable (measured by subtracting the TL condition from its control RL condition). The first model included a dummy variable with two levels which coded whether the transposed/replaced letters were sent to different hemispheres or not, on the assumption that TL priming would be severely reduced when the transposed letters were projected to different hemispheres. The second model included a variable that represented the distance of the position of fixation from the position of transposition. The assumption here was that the priming effect would increase as a function of this latter factor, because the further away the transposed letters are from the fixation location, the less visible they are. Because the magnitude of the priming in addition seemed to vary as a function of the transposition location (i.e., 2-3 vs.4-5), we included a second dummy variable representing the two levels of this variable in both models. The outcomes of the models were (see figure 3 for the predicted values):

$\begin{array}{ll}\text { Model } 1: \text { TLpriming }=42.4^{*}-16.9 \text { Lhem }-14.0 \text { TPplace } & \left(\mathrm{R}^{2}=.288\right) \\ \text { Model } 2: \text { TLpriming }=22.0^{*}+12.1 \text { Dist }^{*}-14.0 \text { TPplace } & \left(\mathrm{R}^{2}=.614\right)\end{array}$

in which: TLpriming $=$ the difference between the RL and TL conditions from Table 2, Lhem $=$ whether or not the transposed/replaced letters were sent to different hemispheres (coded as 1 or 0$)$, Dist $=$ the distance in letter positions between the fixation location and the transposed/replaced letters (ranging from 0 to 3) TPplace $=$ the place of the transposition/replacement ( 23 or 45 , coded respectively as 0 and 1 ), $*=p<$ .05 .

In order to compare the two models, we calculated the likelihood ratio $\lambda$ (Dixon, 2003). This amounted to 21.4, indicating that the second model was 21.4 times more likely as an explanation of the observed priming effects than the first model. Dixon (2003) recommends that $\lambda$-values higher than 10 can safely be interpreted. Model 2 remained significantly more likely than model 1 if the variable TPplace was omitted $\left(\mathrm{R}^{2}\right.$ $=.465$ vs. $\left.\mathrm{R}^{2}=.139, \lambda=10.8\right)$. In addition, the first model not only explained 
significantly less variance, its core variable (Lhem) also failed to reach significance, whereas the core variable of the second model (Dist) did.

\section{Discussion}

Experiment 1 revealed two main findings. First, there was a mean TL-priming effect of $32 \mathrm{~ms}$ for the six-letter target words. Second, the TL-priming effect depended on the distance of the transposed letter pair from the fixation location. The conditions in which the letters at positions 2 and 3 of the six-letter words were transposed showed priming effects ranging from $25 \mathrm{~ms}$ when the words were fixated between the second and the third letter to $62 \mathrm{~ms}$ when the words were fixated between the fifth and the sixth letter. Similarly, the TL-priming effect in the conditions in which letters at positions 4 and 5 were modified caused effects ranging from $0 \mathrm{~ms}$ when the words were fixated between the letters 5 and 6 to $54 \mathrm{~ms}$ when the words were fixated between the letters 2 and 3. This indicates that letter positions are encoded more precisely around the fixation location than away from it. The drop in precision seems to be approximately linear over the range examined in Experiment 1.

An intriguing finding in Figure 3 is why the minimum priming effect was $25 \mathrm{~ms}$ for TL-primes at positions 2-3 (tewlve-TWELVE vs. tuslve-TWELVE) against $0 \mathrm{~ms}$ for TL-primes at positions 4-5 (twevle-TWELVE vs. twemde-TWELVE), as captured by the variable TPplace in the regression analyses. One suggestion could be that this is due to the left-right seriality in visual word recognition. ${ }^{4}$ Given that the primes are presented briefly, in some cases it might be that they cannot be processed fully before they are overwritten by the target. This would be particularly the case when the primes are fixated towards the end. In that situation, quite some time is needed to invert the retina-based activation gradient (see the introduction), so that it is possible that the primes did not get processed completely, but only up to the third letter. In that case no difference is expected between the primes twevle and twemde, because they share the same word beginning. As a result, twevle is not expected to prime the target more than twemde, which is the pattern found in Figure 3.

\footnotetext{
${ }^{4}$ The authors thank Carol Whitney for this suggestion.
} 
The data are not consistent with the late-integration account of the split fovea theory, as proposed by Shillcock et al. (2000). As shown by the model comparison in Figure 3 , the distance between the fixation location and the transposed letters explained significantly more of the variability in the priming effect than whether or not the transposed letters were sent to different hemispheres. The latter would be evidence for Shillcock et al. (2000), as their model predicts different word recognition processes as a function of the fixation location relative to the transposed letters. If the information sent to the cerebral hemispheres was integrated only at a later stage, the response latencies should show a strongly reduced priming effect when the transposed letters were sent to different hemispheres than when they were sent to the same hemisphere (in which case one hemisphere got the correct information and one hemisphere correct information about the letter identities). This is not what we observed; so, the data provide evidence against the late integration account.

A limitation of Experiment 1 is the brief presentation duration of the primes. Perhaps the effect of letter positions depended on visual acuity because the prime letters were presented for only $57 \mathrm{~ms}$ ? To examine this possibility, stimuli were presented until a response was made in Experiment 2, which examined the possible influence of hemifield competitors on visual word recognition.

\section{Experiment 2}

Experiment 2 was designed to further discriminate between the early and the late integration account of the split fovea theory. This experiment also used the lexical decision paradigm, but focused on the effects hemifield competitors may have on visual word recognition.

Recognition of a target word is more difficult when it has to compete against a strong contender. This effect is known in visual word recognition literature as the neighbor frequency effect (Davis \& Taft, 2005; Grainger, O’Regan, Jacobs, \& Segui, 1992; Perea \& Pollatsek, 1998). For instance, Perea and Pollatsek (1998) showed that it takes longer to read the word plate (which has the higher frequency neighbor place) than the control word spoon (which has no higher frequency neighbors). Davis and Taft 
(2005) showed a similar time cost for the word truce relative to sniff, due to the similarity of truce to the high frequency word true.

In Experiment 2, we looked whether we could find a similar inhibition effect due to the presence of high frequency hemifield competitors. These are words that compete with the target word on the basis of the input sent to one hemisphere. For instance, the word plate will compete with the target word place in the $\mathrm{RH}$ when the latter is fixated between the letters 3 and 4, because the LVF input pla- is compatible with both plate and place. In contrast, plate will not be a LVF competitor of place if the word is fixated between the letters 4 and 5 , because the input plac-is not compatible with the word plate.

The stimuli we used in Experiment 2 were six-letter words that differed in the presence of hemifield competitors according to whether they were fixated between letters 2 and 3 or letters 4 and 5. For example, the word in-come has strong (i.e. highfrequency) competitors both in LVF and in RVF when it is fixated between letters 2 and 3: in LVF the word in-deed is a competitor and in RVF be-come is a competitor. In contrast, if the word inco-me is fixated between letters 4 and 5, it does not have any LVF competitors, as there are no other six-letter words in English starting with inco-. A different pattern of competitors is present for the word behalf. It has no RVF competitors when fixated between letters 2 and 3, as there are no other words ending in -half. In contrast, the word beha-ve is a LVF competitor and the word itse-lf is a RVF competitor when beha-lf is fixated between the letters 4 and 5. Finding an effect due to the presence of hemifield competitors would strongly point in the direction of the late integration account; failing to find such an effect would be more in line with the early integration account.

\section{Method}

\section{Participants}

Twenty-one undergraduates from Royal Holloway, University of London took part in this experiment. There were 18 female and 3 male participants. All were native English speakers, had normal or corrected-to-normal vision and were unaware of the 
purpose of the experiment. Further, they were free from any known reading impairments. Eleven students received course credits in return for their participation, and ten of them were paid. All participants were righthanded.

\section{Stimuli and design}

The stimuli of this experiment were 120 words, 120 nonwords and 30 digits. The digits between 1 and 9 had the same function as in the first experiment: they ensured that the participants were fixating between the vertically aligned lines in the centre of the screen at the moment the stimulus appeared. The words were selected by using the English Lexicon Project (Balota et al., 2002) and the Wordgen software (Duyck et al., 2004). They were all six-letter words and were monomorphemic. The nonwords were selected from the nonword list used in the first experiment. They were matched with the total sample of words on neighborhood size $(t<1)$.

The targets were presented on the screen in uppercase between the two vertically aligned lines in the centre of the screen. The six-letter words and nonwords could appear at two different locations relative to the fixation lines, so that they were fixated either between the second and the third letter, or between the fourth and the fifth letter.

There were two different sets of words, because it turned out to be very difficult to find many words like income and behalf, discussed above. The first set of 60 words were of the income and behalf type. Half of them had no LVF competitors when fixated between the fourth and the fifth letter (e.g., inco-me), but did have competitors for both hemispheres when fixated between letters 2 and 3 (e.g., in-deed and be-come). The other half of them had no RVF competitors when fixated between the second and the third letter (e.g., be-half), but did have competitors for both hemispheres when fixated between the letters 4 and 5 (e.g., beha-ve and itse-lf). In other words, at one of the fixation positions, they were fully determined by the information sent to one hemisphere (Figure 4). These stimuli were part of a $2 \times 2$ design (fixation position $\mathrm{x}$ type of word).

Insert Figure 4 about here 
A hemifield competitor was defined as a six-letter, monomorphemic word with a higher frequency than the target word, that could be activated on the basis of the information sent to one hemisphere. In addition, the target words did not have a five- or seven-letter hemifield competitor with a frequency above 7 per million. The frequency estimates were derived from the N-Watch software (Davis, 2005). The stimuli of the two conditions were matched with respect to summed log bigram frequency of the ambiguous part of the word $(t<1)$ and had the same number of competitors that were a neighbour of the target (see Table 3).

Insert Table 3 about here

The second set of 60 words contrasted words without LVF competitors against words with such competitors. Half of these words had no LVF competitor when fixated between the letters 4 and 5 (e.g., tale-nt) but had competitors in both hemispheres when fixated between the letters 2 and 3 (e.g., ta-rget and si-lent for ta-lent). They were compared to 30 control words that had competitors at both fixation locations (e.g., street and du-ring for st-ring, and stri-ke and duri-ng for stri-ng). Experimental and control words were matched on frequency, summed log bigram frequency of the ambiguous part of the word, neighborhood size and amount of morphemes $(t \mathrm{~s}<1$, see Table 3). These stimuli also made up a 2 x 2 design (fixation position $\mathrm{x}$ type of word).

All targets were presented once at the two different fixation positions. To avoid a participant having to evaluate the same stimulus twice, two lists of stimuli were made and equally distributed across the participants. The full set of stimuli can be found in the Appendix appended to the electronic version of this article.

\section{Procedure}

Stimuli were presented in white letters on a black background, using Courier New font, size 12. DMDX software (Forster \& Forster, 2003), CRT displays and 
response boxes were used to present the stimuli and collect the reaction times and error rates. Subjects were sitting at a viewing distance of approximately $60 \mathrm{~cm}$. They were asked to fixate the gap between the two vertically aligned lines that were presented at the onset of each trial in the center of the screen during the total duration of the trial. As mentioned before, digits between 1 and 9 were presented at random times for $80 \mathrm{~ms}$ in the gap between these lines, followed by a mask consisting of an ASCII code 35 (\#). The subjects were told that fixating the centre of the screen as soon as the vertical lines appeared was necessary to be able to evaluate the digits and that making an error on more than 10 digit trials would force the experimenter to exclude their data or to pay them less.

Before the actual experiment started, twelve practice trials were presented on the screen, consisting of two digits, five word stimuli and five nonword stimuli that did not return in the experimental phase. The two centrally presented vertical lines served as the fixation point at the beginning of each trial. After $500 \mathrm{~ms}$, the stimulus appeared between these lines. The position of its appearance was dependent on the condition. The instructions told the participants to press the right button if the target was a word or an even number, or the left button in the case of a nonword and an odd number (the response matching was reversed for one left-handed participant). RT measurement started at word onset. Targets were displayed until a response was made. The intertrial interval was $1 \mathrm{~s}$ between the response and the onset of the next trial.

The experiment lasted approximately 20 minutes and included three breaks after each block of 60 trials.

\section{Results}

On average, 2.75 digits in a total of 30 were missed or evaluated incorrectly by the participants, ensuring that the subjects fixated the gap between the vertical lines when the stimuli appeared. Errors (7\% of the data for word targets) and RTs less than $300 \mathrm{~ms}$ or greater than $1500 \mathrm{~ms}(0.6 \%$ of the data for word targets) were excluded from further analyses. Four targets (blight, dilute, muzzle, and roster) were excluded, because the percentage of errors made on them was more than $25 \%$. This had no effect on the 
matching criteria used in the different conditions. One participant had to be excluded because of a high error rate $(26.7 \%)$, relative to the other participants.

For the stimuli in Set 1, subjects and items analyses of variance (ANOVAs) were carried out based on a 2 (Fixation position: first vs. second) x 2 (Word type: no hemifield competitor at fixation position 1 vs. no hemifield competitor at fixation position 2) design. Table 4 shows the mean reaction times and percentages of errors of the data for word targets. The ANOVA on the latency data yielded a significant main effect of fixation position $[F 1(1,18)=8.95, M S E=4,629.17, p<.01 ; F 2(1,56)=21.38$, $M S E=63,323.29, p<.001]$, with faster mean reaction times to words fixated between the second and third letter (581 ms) compared to words fixated between the fourth and fifth letter $(628 \mathrm{~ms})$. There was no significant interaction between position of fixation and word type $[F 1(1,18)=2.02, M S E=2,766.3, p=.17 ; F 2(1,56)=2.17, M S E=$ $6,438.07, p=.15]$. No effect was significant for the error rates $(p s>.13)$.

Insert Table 4 about here

A similar analysis was run on the data of stimulus set 2 . The latency results replicated the findings of the previous analysis: words fixated between the fourth and the fifth letter were responded to $47 \mathrm{~ms}$ more slowly than words fixated between the second and the third letter $[F 1(1,18)=36.77, M S E=1,252.67, p<.001 ; F 2(1,56)=$ 35.63, $M S E=71,612.91, p<.001]$ and again no interaction was found between fixation location and word type $\left[F_{\mathrm{s}}<1\right]$. So, there was no difference in reaction times between the words that had no LVF hemifield competitors when fixated between the letters 4 and 5 (e.g., tale-nt) and their control stimuli (e.g., stri-ng). The analysis of the error rates yielded a main effect of word type $[F 1(1,18)=14.06, M S E=.002, p<.01]$, with higher mean error rates for targets with no LVF hemifield competitors for fixations between the letters 4 and 5 (tale-nt; 7.0\%) than for the targets with such competitors (e.g., stri-ng; 3.5\%). Importantly, the interaction between word type and fixation position (just like the main effect of fixation position) was not significant ( $p s>.19)$. 


\section{Discussion}

Experiment 2 was conducted to examine the influence hemifield competitors may have on the recognition of foveally presented word stimuli. If the late integration account was correct (as in Shillcock et al.'s, 2000 model of visual word recognition), we expected to observe longer lexical decision times for words with high-frequency hemifield competitors than for words without such competitors. In contrast, failing to find an interaction between fixation position and the presence of hemifield competitors would be evidence for an early integration account (as in the SERIOL model, for example). We tested these hypotheses with two sets of stimuli.

For the first set, the late integration account predicted that words with hemifield competitors in both hemispheres at fixation position 1 (e.g., in-come), but not at fixation position 2 (e.g., inco-me) would be recognized more slowly when fixated between the second and third letter than when fixated between the fourth and fifth letter. Similarly, words with competitors in both hemispheres at fixation position 2 (e.g., beha-lf), but not at fixation position 1 (e.g., be-half) would cause faster identification when fixated between the letters 2 and 3 than when fixated between the letters 4 and 5. This would lead to a significant interaction between type of word and fixation location. In contrast, the early integration account predicted faster latencies at the first fixation location for both word types in line with the overall OVP effect.

For the second set of stimuli, the late integration account again predicted shorter recognition times when there were no competitors in one hemisphere (e.g., tale-nt) than for matched control words with such competitors (e.g., stri-ng). The early integration account predicted shorter latency data for the first fixation position, independently of the existence of hemifield competitors.

The results of Experiment 2 are in line with the early integration account. As in Experiment 1, there was a clear effect of the fixation position, but no interaction with the manipulated word characteristics. When the stimuli were fixated at the beginning of the word, lexical decision times were on average $47 \mathrm{~ms}$ faster than when the fixation was at the end of the word, in line with the OVP effect (Figure 1). However, exactly the same effect was found for all types of words, which means that the type of the word had a negligible effect on the processing of the word. Whether or not the information 
received by a hemisphere unambiguously indicated the identity of the target word had no influence on the time taken to classify that word.

\section{General Discussion}

In two lexical decision experiments, we investigated the consequences of interhemispheric communication for the processing of foveally presented stimuli. Assuming that the split fovea theory is correct (Figure 1), we started from the finding that the input to the left of the fixation location is initially projected to the right cerebral hemisphere, whereas the information to the right of the fixation location is sent to the left hemisphere.

The first experiment used a masked form priming paradigm, in which two adjacent letters of the prime were transposed or replaced. We found that fixation position had a substantial influence on the transposed letter priming effect, but that the influence was not due to the fact whether or not the letters were sent to different hemispheres. In the second experiment, stimuli were presented until a response was made and were fixated in such a way that the input to one hemisphere either uniquely pointed to the target word or was ambiguous (i.e., consistent with another word of a higher frequency). Again, fixation position influenced the results significantly, but there was no effect due to the presence of hemifield competitors.

We conducted these experiments in an attempt to test predictions made by the late integration account embodied in the model of Shillcock et al. (2000). In this model, the two cerebral hemispheres operate independently in the early processes of word recognition and share information only at a relatively late stage of processing. We hypothesized that such a model would predict a stronger transposed letter priming effect if two transposed letters were projected to the same hemisphere (e.g., tew-lve) than if they were projected to different hemispheres (e.g., te-wlve). In the former case, each hemisphere has the correct letters to activate the relevant word candidate (e.g., twe-lve). Only the letter positions in one hemifield are transposed, which in Shillcock et al.'s (2000) model are less important, due to its coarse coding assumption. In contrast, fixation between the two transposed letters (e.g., te-wlve) sends the transposed letters to 
the 'wrong' hemisphere, making it more difficult to activate the target word. For example, when fixating between the second and third letter of cuople (the TL prime at position 2-3 of the target word COUPLE), the first two letters $\mathrm{cu}$ - are more likely to activate words like cuddle, cursed, or custom than couple, and the letters at the right side of the fixation (i.e. -ople) are more likely to activate the word people than the target.

In order to control for the confound of reduced visual acuity when the transposed letters are not at the center of the visual field, we had all words fixated at five different locations in Experiment 1. This turned out to be a very good control indeed, because the transposed letter priming effect was much better explained by assuming that the magnitude of the effect depended on the distance of the transposed letters from the position of fixation than on whether or not the transposed letters were projected to the same hemisphere (Figure 3). Therefore, the more parsimonious explanation of the observed transposed letter priming effects is that they depend on the acuity with which the letter positions have been encoded and not on whether the letters have been projected to the 'correct' hemisphere.

A second, independent test of the late integration account was performed in Experiment 2. By comparing words with and without hemispheric competitors (e.g., income has in-deed and be-come as RH and LH competitors, whereas inco-me has no competitors in the $\mathrm{RH}$ ), we aimed to determine if there was any evidence for parallel intrahemispheric word processing in the early stages of visual word recognition. In this experiment, stimuli were presented until a response was made, allowing us to rule out the possibility that the absence of evidence for the late integration account in Experiment 1 was somehow related to the brief presentation of the primes. Once again, however, we failed to find evidence for late integration. We did not observe a neighbor frequency effect (e.g., Davis \& Taft, 2005; Perea \& Pollatsek, 1998) as a result of the inhibitory influence hemifield competitors could have on the recognition of the target word. Data only differed as a function of fixation position, with faster recognition times when words were fixated at the beginning than at the end, in line with the OVP effect.

The findings of Experiments 1 and 2 point against Shillcock et al.'s (2000) late integration account of interhemispheric communication in foveal word recognition. In contrast, they are fully in line with the early integration account, as in Whitney's (2001) 
SERIOL model. According to this model, all information is first converged in the dominant hemisphere before word processing starts. This model predicts that for left dominant participants (as we can assume all our participants were), word processing will be faster after fixations on the left half of the word than after fixations on the right half, a result we found in both of our experiments. The model further predicts an absence of effects due to whether or not transposed letters are sent to the same hemisphere and whether or not there are hemifield competitors, as again observed in our experiments.

These data are in line with the observation in brain imaging research that a visual word form area in the occipito-temporal area of the language-dominant cerebral hemisphere becomes active in word processing irrespective of the retinal position of the word (Cai et al., 2008; Cohen et al., 2000; Liu et al., 2008; Vigneau et al., 2005). Our data strongly suggest that the processing in this word form area is indeed what determines the time needed to make a lexical decision. In line with the early integration account, the information received by the non-dominant hemisphere must be transmitted to the dominant hemisphere before word processing starts. An intriguing question at this point is whether in parallel some word processing may be going on in the non-dominant hemisphere without having an impact on the lexical decision times. As suggested by Pernet et al.'s (2007) MEG study, an asymmetry of activation in the occipito-temporal cortex does not imply complete absence of activity in the non-dominant hemisphere. One way to investigate this issue would be to apply transcranial magnetic stimulation to the homologue of the word form area in the non-dominant hemisphere and see what consequences this has (see Stewart, Meyer, Frith, \& Rothwell, 2001, for such a paradigm and some suggestive preliminary evidence).

The degree of laterality of visual word recognition is further interesting because a very similar issue exists in speech processing. According to one of the dominant models (Hickok \& Poeppel, 2004) word recognition in speech perception involves auditory-responsive fields in the superior temporal gyrus bilaterally. From there the signal diverges into two processing streams, a ventral stream, which is involved in mapping sound onto meaning, and a dorsal stream, which is involved in mapping sound onto articulatory-based representations. In Hickok and Poeppel's (2004) view the ventral stream operates bilaterally with a modest degree of left dominance. In contrast, 
other authors report evidence for a larger degree of lateralization in speech recognition (e.g., Friederici \& Alter, 2004; Shtyrov, Pihko, \& Pulvermüller, 2005). Cai et al. (2008) further argued that the right dominance of the occipito-temporal cortex in French participants with speech production in the right frontal cortex, can only be explained by strong interactions between the word reading system and the speech output system. There are no other reasons why the word form area in these people would be lateralized to the right, given that French is a language read from left to right, so that the information in RVF is much richer (containing most of the fixated word and the next word) than the information in LVF (containing but the beginning of the presently fixated word). This clearly is an issue for further research, ideally be comparing the brain activity of participants with left and right language dominance, along the lines introduced by Hunter et al. (2007) and Cai et al. (2008). 


\section{References}

Balota, D.A., Cortese, M.J., Hutchison, K.A., Neely, J.H., Nelson, D., Simpson, G.B., Treiman, R. (2002). The English Lexicon Project: A web-based repository of descriptive and behavioral measures for 40,481 English words and nonwords. http://elexicon.wustl.edu/, Washington University.

Brysbaert, M. (1994). Interhemispheric transfer and the processing of foveally presented stimuli. Behavioural Brain Research, 64, 151-161.

Brysbaert, M. (2004). The importance of interhemispheric transfer for foveal vision: A factor that has been overlooked in theories of visual word recognition and object perception. Brain and Language, 88, 259-267.

Brysbaert, M., \& Nazir, T. (2005). Visual constraints in written word recognition: evidence from the optimal viewing-position effect. Journal of Research in Reading, 28, 216-228.

Brysbaert, M., Vitu, F., \& Schroyens, W. (1996). The right visual field advantage and the optimal viewing position effect: On the relation between foveal and parafoveal word recognition. Neuropsychology, 10, 385-395.

Bunt, A.H., Minckler, D.S., \& Johanson, G.W. (1977). Demonstration of bilateral projection of the central retina of the monkey with horseradish peroxidase neuronography. Journal of Comparative Neurology, 171, 619-630.

Cai, Q., Lavidor, M., Brysbaert, M., Paulignan, Y., \& Nazir, T.A. (2008). Cerebral lateralization of frontal Lobe language processes and lateralization of the posterior visual word processing system. Journal of Cognitive Neuroscience, 20, $1-10$.

Cohen, L., Dehaene, S., Naccache, L., Lehericy, S., Dehaene-Lambertz, G., Henaff, M.A., \& Michel, F. (2000). The visual word form area: Spatial and temporal characterization of an initial stage of reading in normal subjects and posterior split-brain patients. Brain, 123, 291-307.

Davis, C.J. (2005). N-Watch: A program for deriving neighborhood size and other psycholinguistic statistics. Behavior Research Methods, 37, 65-70. 
Davis, C.J., \& Taft, M. (2005). More words in the neighborhood: Interference in lexical decision due to deletion neighbors. Psychonomic Bulletin \& Review, 12, 904910.

Dixon, P. (2003). The p-value fallacy and how to avoid It. Canadian Journal of Experimental Psychology, 57, 189-202.

Duyck, W., Desmet, T., Verbeke, L., \& Brysbaert, M. (2004). Wordgen: A tool for word selection and non-word generation in Dutch, English, German, and French. Behavior Research Methods, Instruments \& Computers, 36, 488-499.

Ellis, A.W. (2004). Length, formats, neighbours, hemispheres, and the processing of words presented laterally or at fixation. Brain and Language, 88, 355-366.

Ellis, A.W., Brooks, J., \& Lavidor, M. (2005). Evaluating a split fovea model of visual word recognition: effects of case alternations in the two visual fields and in the left and right halves of words presented at the fovea. Neuropsychologia, 43, 1128-1137.

Forster, K.I., Davis, C., Schoknecht, C., \& Carter, R. (1987). Masked priming with graphemically related forms: Repetition or partial activation? Quarterly Journal of Experimental Psychology, 39, 211-251.

Forster, K.I., Forster, J.C. (2003). DMDX: A Windows display program with millisecond accuracy. Behavior Research Methods, Instruments \& Computers, $35,116-124$.

Friederici, A.D. \& Alter, K. (2004). Lateralization of auditory language functions: A dynamic dual pathway model. Brain and Language, 89, 267-276.

Grainger, J., O’Regan, J.K., Jacobs, A.M., \& Segui, J. (1992). Neighborhood frequencyeffects and letter visibility in visual word recognition. Perception \& Psychophysics, 51, 49-56.

Hickok, G. \& Poeppel, D. (2004). Dorsal and ventral streams: A framework for understanding aspects of the functional anatomy of language. Cognition, 92, 6799.

Hunter, Z.R. \& Brysbaert, M. (2008). Visual half-field experiments are a good measure of cerebral language dominance if used properly: Evidence from fMRI. Neuropsychologia, 46, 316-325. 
Hunter, Z.R., Brysbaert, M., \& Knecht, S. (2007). Foveal word reading requires interhemispheric communication. Journal of Cognitive Neuroscience, 19, 13731387.

Knecht, S., Dräger, B., Deppe, M., Bobe, L., Lohmann, H., Floel, A., et al. (2000). Handedness and hemispheric language dominance in healthy humans. Brain, $123,2512-2518$.

Lavidor, M., \& Walsh, V. (2004a). The nature of foveal representation. Nature Reviews Neuroscience, 5, 729-735.

Lavidor, M., \& Walsh, V. (2004b). Magnetic stimulation studies of foveal representation. Brain \& Language, 88, 331-338.

Lavidor, M., Ellis, A.W., Shillcock, R., \& Bland, T. (2001). Evaluating a split processing model of visual word recognition: effects of word length. Cognitive Brain Research, 12, 265-272.

Lavidor, M., Ellison, A., \& Walsh, V. (2003). The cortical representation of centrally presented words: A magnetic stimulation study. Visual Cognition, 10, 341-362.

Lavidor, M., Hayes, A., Shillcock, R., \& Ellis, A.W. (2004). Evaluating a split processing model of visual word recognition: Effects of orthographic neighborhood size. Brain and Language, 88, 312-320.

Leff, A. (2004). A historical review of the representation of the visual field in primary visual cortex with special reference to the neural mechanisms underlying macular sparing. Brain and Language, 88, 268-278.

Liu, C., Zhang, W.T., Tang, Y.Y., Mai, X.Q., Chen, H.C., Tardif, T., \& Luo, Y.J. (2008). The visual word form area: Evidence from an fMRI study on the implicit processing of Chinese characters. NeuroImage, 40, 1350-1361.

Martin, C.D., Thierry, G., Démonet, J.F., Roberts, M., \& Nazir, T. (in press). ERP evidence for the split fovea theory. Brain Research.

Masson, M.E.J. \& Loftus, G.R. (2003). Using confidence intervals for graphically based data interpretation. Canadian Journal of Experimental Psychology, 57, 203-220.

O’Regan, J.K., \& Jacobs, A.M. (1992). Optimal viewing position effect in word recognition: A challenge to current theory. Journal of Experimental Psychology: Human Perception and Performance, 18, 185-197. 
Perea, M., \& Lupker, S.J. (2003). Transposed-letter confusability effects in masked form priming. In S. Kinoshita, \& S.J. Lupker (Eds.), Masked priming: The state of the art. Hove, UK: Psychology Press.

Perea, M., \& Pollatsek, A. (1998). The effects of neighborhood frequency in reading and lexical decision. Journal of Experimental Psychology: Human Perception and Performance, 24, 767-779.

Pernet, C., Uusvuori, J., \& Salmelin, R. (2007). Parafoveal-on-foveal and foveal word priming are different processes: Behavioral and neurophysiological evidence. NeuroImage, 38, 321-330.

Portin, K., Salenius, S., Salmelin, R., \& Hari, R. (1998). Activation of the human occipital and parietal cortex by pattern and luminance stimuli: neuromagnetic measurements. Cerebral Cortex, 8, 253-260.

Raaijmakers, J.G.W., Schrijnemakers, J.M.C., \& Gremmen, F. (1999). How to deal with "The language-as-fixed-effect fallacy": Common misconceptions and alternative solutions. Journal of Memory and Language, 41, 416-426.

Reinhard, J. \& Trauzettel-Klosinski, S. (2003). Nasotemporal overlap of retinal ganglion cells in humans: A functional study. Investigative Ophthalmology \& Visual Science, 44, 1568-1572.

Schoonbaert, S., \& Grainger, J. (2004). Letter position coding in printed word perception: Effects of repeated and transposed letters. Language and Cognitive Processes, 19, 333-367.

Shillcock, R., Ellison, T.M., \& Monaghan, P. (2000). Eye-fixation behavior, lexical storage, and visual word recognition in a split processing model. Psychological Review, 107, 824-851.

Shtyrov, Y., Pihko, E., \& Pulvermuller, F. (2005). Determinants of dominance: Is language laterality explained by physical or linguistic features of speech? NeuroImage, 27, 37-47.

Stewart, L., Meyer, B.U., Frith, U., \& Rothwell, J. (2001). Left posterior BA37 is involved in object recognition: a TMS study. Neuropsychologia, 39, 1-6.

Tootell, R.B.H., Switkes, E., Silverman, M.S., \& Hamilton, S.L. (1988). Functional anatomy of macaque striate cortex. II. Retinotopic organization. The Journal of Neuroscience, 8, 1531-1568. 
Vigneau, M., Jobard, G., Mazoyer, B., \& Tzourio-Mazoyer, N. (2005). Word and nonword reading : What role for the visual word form area ? NeuroImage, 27, 694705.

Whitney, C. (2001). How the brain encodes the order of letters in a printed word: The SERIOL model and selective literature review. Psychonomic Bulletin \& Review, $8,221-243$. 


\section{Acknowledgements}

This research was made possible by a Socrates exchange between Royal Holloway, University of London and Ghent University, and by the ESRC grant RES-000-22-2662 "How do anatomical constraints affect visual word recognition?". 
TABLE 1

Mean Values of the Matching Criteria in Experiment 1

\begin{tabular}{ccccc}
\hline Condition & $\begin{array}{c}\text { Example } \\
\text { (Target TWELVE) }\end{array}$ & Length & Bigram & Trigram \\
\hline TL prime 2-3 & tewlve & 6 & 1.92 & 0.74 \\
RL prime 2-3 & tuslve & 6 & 1.92 & 0.73 \\
\cline { 2 - 5 } TL prime 4-5 & twevle & 6 & 1.98 & 0.73 \\
RL prime 4-5 & twemde & 6 & 1.97 & 0.75 \\
\hline Note TL Transposed Letter; RL = Replaced Letter; Bigram $=$ mean log Bigram token
\end{tabular}

Note. $\mathrm{TL}=$ Transposed Letter; RL $=$ Replaced Letter; Bigram $=$ mean log Bigram token frequency; Trigram $=$ mean log Trigram token frequency. 
TABLE 2

Mean Response Times in Milliseconds (and Error rates in Percentage) for Word Targets in Experiment 1

\begin{tabular}{cccccc}
\hline & \multicolumn{5}{c}{ Fixation location } \\
\cline { 2 - 6 } Prime type \& position & 1 & 2 & 3 & 4 & 5 \\
\hline Transposed 2-3 & $595(4.6)$ & $572(3.9)$ & $570(2.1)$ & $589(4.6)$ & $604(5.3)$ \\
Replaced 2-3 & $623(3.9)$ & $597(3.5)$ & $615(5.6)$ & $624(6.7)$ & $666(9.1)$ \\
Transposed 4-5 & $590(3.7)$ & $570(3.0)$ & $577(3.7)$ & $603(5.6)$ & $661(5.4)$ \\
Replaced 4-5 & $629(7.2)$ & $624(5.8)$ & $598(3.7)$ & $615(4.2)$ & $660(6.7)$ \\
\hline
\end{tabular}


TABLE 3

Mean Values of the Matching Criteria in Experiment 2. Numbers indicate the mean values of the hemifield competitors for the respective critical conditions.

\begin{tabular}{llcccccc}
\hline & Condition & Example & Length & Morpheme & Frequency & Bigram & $\mathrm{N}$ \\
\hline \multirow{2}{*}{ Set 1 } & HFC for fixation 1 & INCOME & 6 & 1 & & 8.36 & 10 \\
& HFC for fixation 2 & BEHALF & 6 & 1 & & 8.24 & 10 \\
\cline { 2 - 8 } Set 2 & HFC for fixation 1 & TALENT & 6 & 1 & 7.67 & 8.49 & 1.20 \\
& HFC for fixation 1+2 & STRING & 6 & 1 & 7.72 & 8.70 & 1.03 \\
\hline
\end{tabular}

Note. HFC = Hemifield Competitor; Bigram = summed log Bigram frequency of the ambiguous word part; $\mathrm{N}=$ number of competitors that are an orthographic Neighbour of the target in Set 1, orthographic Neighbourhood size in Set 2. 
TABLE 4

Mean Response Times in Milliseconds (and Error rates in Percentage) for Word Targets in Experiment 2

\begin{tabular}{clcc}
\hline & \multicolumn{2}{c}{ Word Type } & \multicolumn{2}{c}{ Fixation Location } \\
\cline { 3 - 4 } & & 1 & 2 \\
\hline \multirow{2}{*}{ Set 1 } & HFC for Fixation Location 1 (e.g., INCOME) & $552(4.6)$ & $615(7.7)$ \\
& HFC for Fixation Location 2 (e.g., BEHALF) & $609(7.7)$ & $640(7.0)$ \\
\cline { 2 - 4 } Set 2 & HFC for Fixation Location 1 (e.g., TALENT) & $568(6.3)$ & $620(7.7)$ \\
& HFC for Fixation Location 1+2 (e.g., STRING) & $571(2.8)$ & $614(4.2)$ \\
\hline
\end{tabular}

Note. $\mathrm{HFC}=$ Hemifield Competitor 


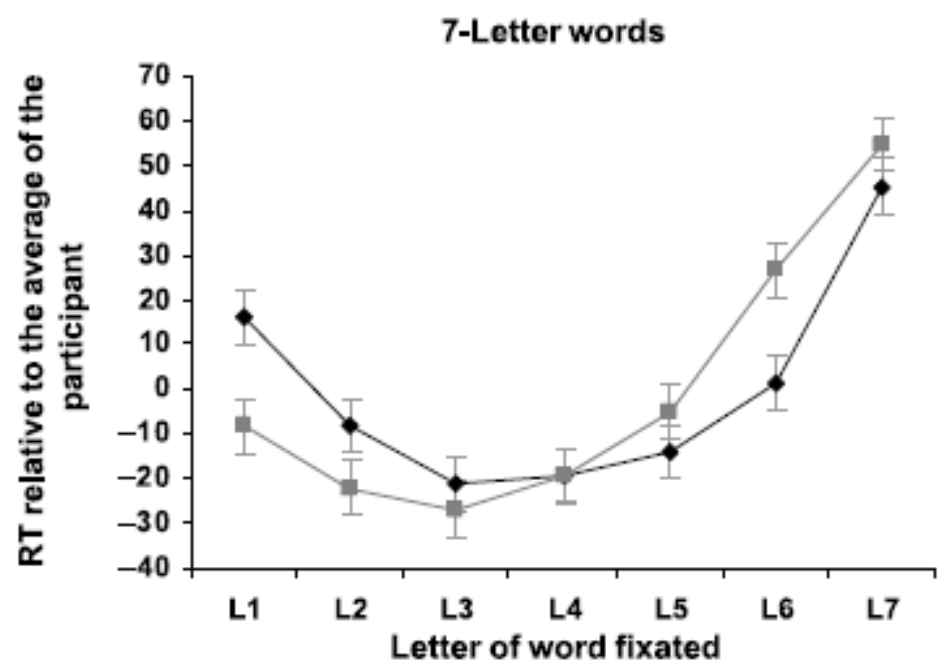

FIGURE 1. OVP curves for the naming of seven-letter words for participants with left hemisphere dominance (light gray line) and participants with right hemisphere dominance (dark gray line). Left dominant participants were faster than right dominant participants to name the words after fixation on the word beginning, whereas the reverse was true for fixation on the word end. This is because stimuli presented in RVF have direct access to the left hemisphere, whereas stimuli presented in LVF have direct access to the right hemisphere. (Data from Hunter, Brysbaert, \& Knecht, 2007) 


\section{Fixation position}

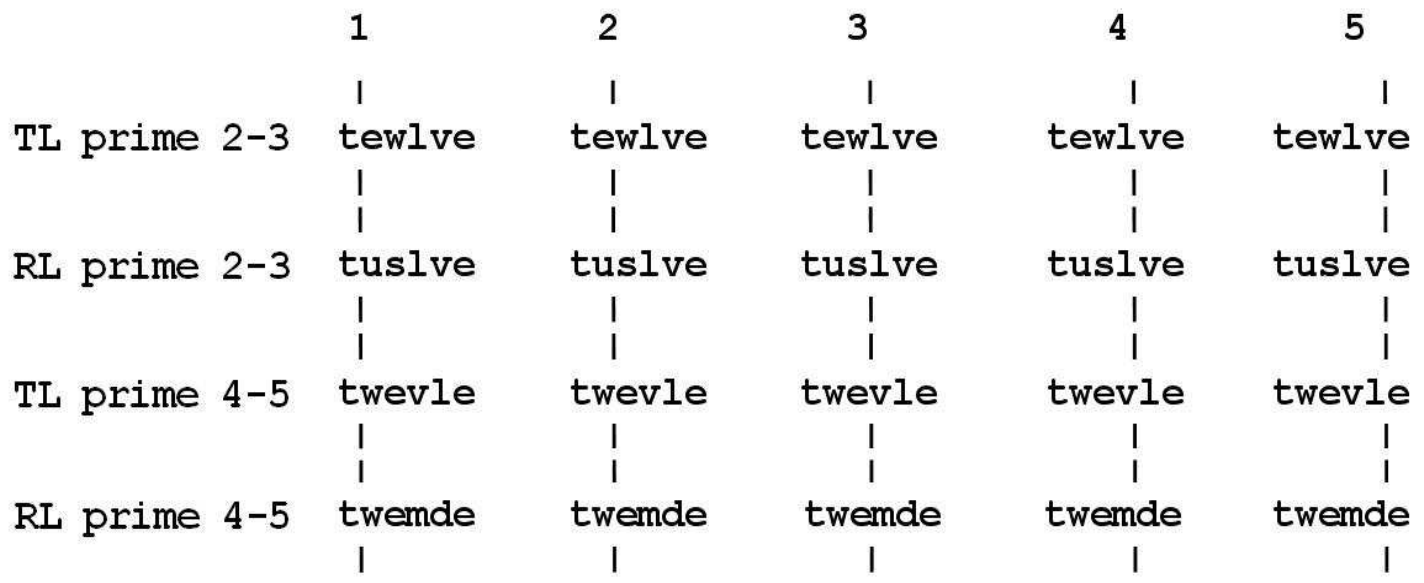

FIGURE 2. Examples of the TL and RL primes in Experiment 1, for the target word TWELVE, together with the five fixation positions that were used. 
Model 1 Position 2-3

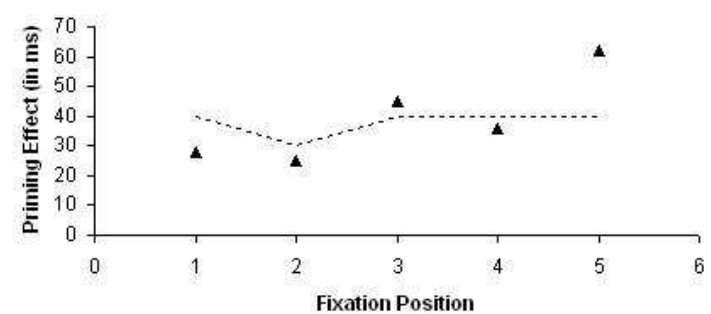

Model 1 Position 4-5

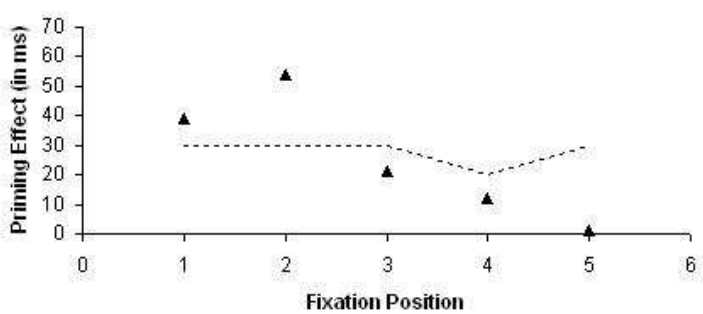

Model 2 Position 2-3

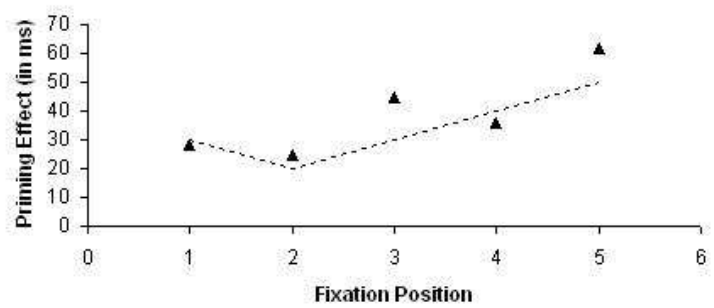

Model 2 Position 4-5

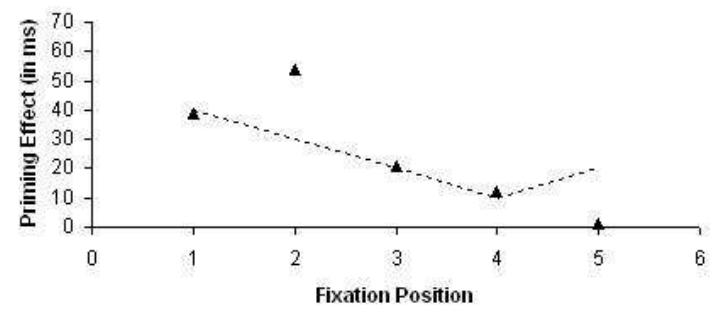

FIGURE 3. Model comparisons for the priming effect of the TL-primes compared to the RLprimes at positions 2-3 and 4-5 of the word targets used in Experiment 1. Model 1 predicts diminished priming only when the transposed letters are sent to the wrong hemispheres. Model 2 predicts diminished priming for transposed letters close to the fixation location, independent of whether the transposed letters are sent to the same or to different hemispheres. 
Fixation position

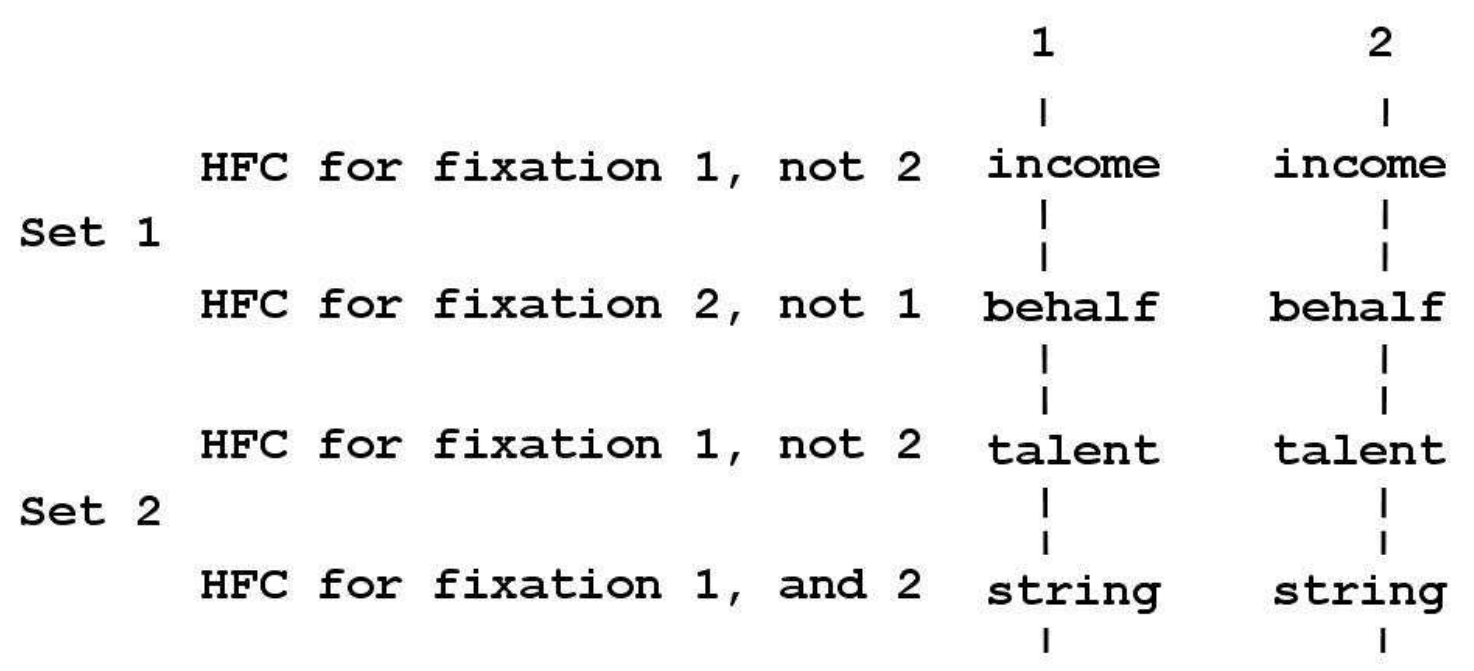

FIGURE 4. Example stimuli for the four word types and two fixations positions in Experiment 2.

Note. HFC $=$ Hemifield Competitor 